\title{
Involve Me And I Will Understand!-Abstract Data Visualization In Immersive Environments
}

\author{
René Rosenbaum, Jeremy Bottleson, Zhuiguang Liu and Bernd Hamann \\ Institute of Data Analysis and Visualization (IDA) \\ Department of Computer Science \\ University of California, Davis, CA 95616, U.S.A.
}

\begin{abstract}
Literature concerning the visualization of abstract data in immersive environments is sparse. This publication is intended to (1) stimulate the application of abstract data visualization in such environments and to (2) introduce novel concepts involving the user as an active part of the interactive exploratory visualization process. To motivate discussion, requirements for the visualization of abstract data are reviewed and then related to the properties of immersive environments in order to show its potential for data visualization. This leads to the introduction of a novel concept for immersive visualization based on the involvement of the viewers into the data display. The usefulness of the concept is shown by two examples demonstrating that immersive environments are far more than tools to create visually appealing data representations.
\end{abstract}

\section{Introduction}

Visualization has been quite successful for data analysis, but limitations still exist. One limiting factor is the widely used two-dimensional (2D) display and interaction technology. Although we live and interact in a three-dimensional (3D) environment, most data visualizations use only two dimensions and neglect depth. This is often reasonable as the display of 3D data on 2D desktop screens imposes many drawbacks, such as occlusion, cumbersome interaction, and missing depth cues.

First attempts to overcome these problems in data visualization were made by presenting data in immersive environments (IE). IEs are able to provide a synthetic 3D display and interaction space, which is rendered in the first-person viewpoint [1]. Due to the use of stereoscopic-vision and motion parallax, IEs are able to mimic our natural $3 \mathrm{D}$ viewing environment and thus can provide a high level of physical immersion (see Figure 1, left). Early IEs relied on costly hardware, but with the recent boost in 3D display hardware, the technology now becomes much more affordable.

Research to visualization in IES mainly focusses on spatial data. Not much is known about abstract data. This might be due to the fact that the "natural" representation of available spatial components is often considered to be the

\footnotetext{
* The author gratefully acknowledges the support of Deutsche Forschungsgemeinschaft
} (DFG) for funding this research (\#RO3755/1-1) 
main reason for its application. Although not necessarily containing spatial components, abstract data must also be spatially arranged in order to be displayed. Thus, abstract data visualization can benefit from IEs [1].

We would like to review and stimulate discussion on the visualization of abstract data in immersive environments - immersive Abstract Data Visualization (iAV). We also introduce a novel concept for iAV not available for non-immersive viewing technology. After reviewing existing research in this field (Section 2), the main requirements of the visualization of abstract data are discussed (Section 3) and used to show that the properties of IEs can help to advance data representation and interactive analysis (Section 4). To illustrate the mainly undiscovered potential of iAV, a novel concept considering the user as an active part of the visualization instead of a passive viewer is introduced (Section 5). Its implementation is shown for the two traditional visualization techniques scatter and parallel coordinates plot, each focussing on a different aspect of the concept. We conclude that iAV will not replace common desktop-based data exploration, but will enrich the state of related visualization technology by novel means for visual representation and the way we interact with the data (Section 6).
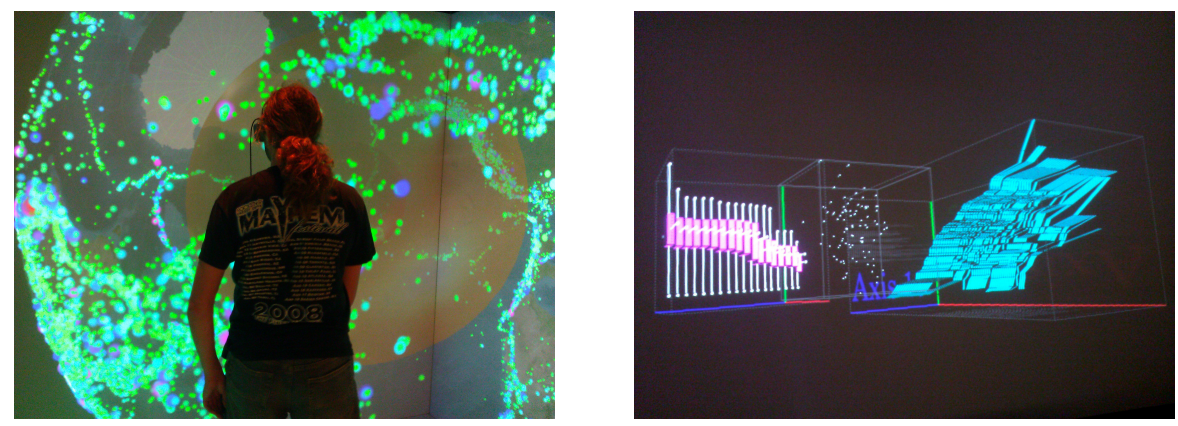

Fig. 1. The visualization of spatial data in immersive environments has a long tradition (left). Visualization of abstract data in such environments is usually limited to threedimensional adaptations of two-dimensional data displays (right).

\section{Work Related to Immersive Abstract Data Visualization}

Compared to the immersive visualization of spatial data, often referred to as scientific data, not much is known about iAV. Existing approaches can be mainly subdivided into two groups - strategies with or without using the virtual world metaphor.

Approaches applying the virtual world metaphor use visuals that mimic principles and behavior of the real world. Most strategies create a "virtual world" 
from the data to facilitate conveyance of data aspects and to simplify navigation. Others enrich a virtual world by Infographics [2]. The concept introduced in this paper does not take advantage of a virtual world metaphor and thus is significantly different from this approach.

Visualizations for IEs not using the virtual world metaphor are sole data displays. They take advantage of the provided depth cues and thus an enriched and more natural 3D data representation. Most of the techniques are founded on developments for desktop environments, such as the techniques shown in Figure 1, right. Recent research [1] has shown that this provides a much better data representation and supports conveyance of properties of the data. Most common are traditional scatter plots that can be found in all varieties $[3,4$, 1] often paired with an appropriate glyph-based representation to show more than three data dimensions in a single display. However, adaptations of other data displays, such as line charts [3] or the parallel coordinates plot [4], have also been proposed. Novel visualization strategies specifically developed for immersive environments are rare. The few approaches have mainly been proposed for single application areas and data types that have specific needs, such as software [5] or documents [6]. These solutions are strongly domain- and problem-specific. The novel concept introduced in this paper is broadly applicable and involves the user into the representation. Thus, it opens up for a whole new view of iAV.

\section{Main Requirements for Abstract Data Visualization}

The visualization of abstract data is related to the field of information visualization. CARD ET AL. [7] identified an appropriate (1) visual representation and (2) means for interaction as the key requirements for meaningful solutions in this domain. We will discuss to what degree these aspects can be implemented by $2 \mathrm{D}$ and $3 \mathrm{D}$ representations.

Visual representation In order to amplify cognition [8], properties of the data are mapped into appropriate perceptual attributes, such as position, orientation, size, shape, or color. Thereby, position is probably the most often used attribute as it relies on the strong ability of the human visual system to determine distances and relate objects to each other. This ability is strongest in $2 D$ representations. Thus, they have a long successful tradition especially for low-dimensional data sources and can reduce visual overload and the complexity of comparison and relation tasks. However, when data becomes more complex, e.g., has higher dimensionality, their limits become quickly apparent. 3D representations have two main advantages compared to their 2D counterparts: (1) there is an additional spatial dimension available for value encoding and (2) they help shift the viewing process from being a cognitive task to being a perception task [9]. This enables a much faster processing of the contents [10] and makes them more natural and appealing to us. Thus, encoding data into a third spatial 
dimension is often superior compared to other means. The ability of the human visual system to determine distances, however, is less accurate in 3D space.

In order to display a data representation on a common screen, it must be projected into the $2 \mathrm{D}$ screen space. This process causes occlusion issues and visual clutter for 3D graphics. Due to this, such representations have been labeled weak-3D [11].

Interaction Most of the established interaction devices are designed for $2 \mathrm{D}$ displays and representations. Widely accepted solutions for interacting with 3D representations on 2D screens are not available making data exploration in such set-ups difficult to impossible. As meaningful interaction is also imperative to solve the inherent occlusion problem and to improve the viewers's understanding of $3 \mathrm{D}$ content, there has always been a strong controversy whether $3 \mathrm{D}$ representations are meaningful in the visualization of abstract data. Given their advantages for visual data representation, one might conclude that the lack of appropriate means for interaction is probably the main reason why $3 \mathrm{D}$ information displays are often neglected.

\section{Benefits of Immersive Environments for Abstract Data Visualization}

In this section, we show that the realistic three-dimensional data representation and intuitive means for interaction inherently provided by IEs are able to meet the requirements for abstract data visualization, and thus allowing full advantageous use of 3D graphics. IEs can also provide a solution for the often corresponding problem of visualization of large data volumes.

\subsection{Realistic Three-dimensional Representation}

IEs create the impression of a user being "present" in the visualization, resulting in a data representation that is natural to the viewer and requires less cognitive strain. This leads to amplified cognition as well as an increased user acceptance [12] and performance in tasks typical for information exploration [13]. First developments in iAV revealed that they are well-suited for tasks requiring a spatial understanding [1] or a mental model of the data and its representation [12]. As shown for simple data or more sophisticated cluster displays as well as tree and graph visualizations, task completion times can often be significantly reduced [14, 12] or are generally lower [15] compared to that of $2 \mathrm{D}$ representations. Keys for the success are the provided depth cues [12] and immersion [15]. However, it has been reported [11] that such a strong-3D display [11] does not always provide better results than a weak-3D display. This especially applies to less cluttered data representations. 


\subsection{Intuitive Means for Interaction}

IEs are closer to natural interaction than many other forms of computer systems and inherently overcome the problems imposed by interacting with $3 \mathrm{D}$ data. They support most tasks relevant to abstract data exploration [13]. Crucial for their success is the natural and effortless ability to change the viewing perspective by head-based rendering (HBR), providing effective means in solving occlusion problems and adjusting the detail level of the data. Panning and zooming may be used to examine different parts of the data representation. As stated in [15], this characteristic leads to better task completion times, higher usefulness ratings, and less disorientation compared to other forms of interaction.

As the viewer is immersed in the environment and can almost "touch" it, more complex forms of interaction such as selection may also be provided in an intuitive and natural way to the viewer. The respective implementation, however, would strongly depend on the given application context and available hardware, such as magic wands or data gloves.

\subsection{Visualization of Large Data Volumes}

Because data must often be displayed in detail and in many different views at a time, the available screen space becomes one of the limiting factors in the visualization of large data volumes. Clusters of computer screens and display walls have recently become very common in pushing the existing boundaries. Immersive environments probably representing the ultimate solution for this trend. They provide a field of view in resolutions that shifts the constraining factor from used hardware to the human visual system's abilities. The literally unlimited screen space also significantly reduces viewing interactions. The supported natural means for zooming and panning help to overcome the general problem of visual overload.

\subsection{Drawbacks}

Despite the many different advantages provided by IEs, it has been reported that initial learning efforts may increase task completion times especially for users unfamiliar with IEs [14]. However, such difficulties are usually quick to overcome [16]. There are also cases where IE usage does not provide a significant advantage compared to non-immersive display technology [11]. As IEs only advance the means for data representation and interaction, this especially applies to solutions that strongly rely on other stages of the visualization process, such as filtering. In poorly designed representations, disorientation or cybersickness may appear [16]. One major drawback of IEs often neglected in related literature is their reliance on a direct interaction with the data. The thereby resulting higher level of physical activity compared to common desktop-based data analysis can lead to fatigue especially for complex and long exploration tasks. 

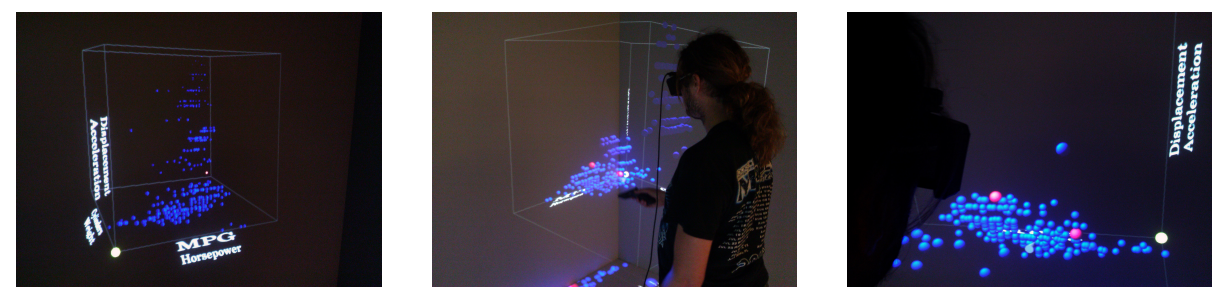

Fig. 2. Immersive Scatter Plots without (left) and with an immersed user looking at one of the visible three-dimensional data clusters (center, right).

\section{Novel Strategies for Immersive Visualization of Abstract Data}

\subsection{The Concept}

This section introduces a novel concept for iAV that introduces new display and interaction strategies in addition to the advantages of IEs for data visualization.

"Tell me and I'll forget. Show me and I may remember. Involve me and I'll understand." - Konfuzius.

The concept is founded on the ability of IEs to let the viewer immerse with the data and aims to gain more insight with less effort. Immersion is used to let the user be an active part of the visualization instead of being a passive viewer opening up a novel display and interaction paradigm. The foundation of the proposed concept is the exceptional support of location changes and spatial understanding in IEs allowing viewers to easily position and relate themselves to the data representation. Considering the viewer as part of the visualization promises to provide a better understanding of the data and intuitive means for interaction. Dependent on the role of the viewer within such a truly immersive visualization, two different approaches are introduced: the user is considered as (1) part of the data that can interact with others or (2) part of the layout that serves as a reference scale or means to change the layout. We are aware of the fact that the proposed concept might require a new understanding of data representation. Involving the user, however, is the next logical step in the development of more sophisticated visualization techniques for IEs.

The implementation of the concept is demonstrated by the extension of two widely used visualization techniques - scatter and parallel coordinates plot. Each implementation is explained focusing on the novel means for visual representation and interaction. The techniques have been tested in the UC Davis KeckCAVES virtual reality environment consisting of a four-walled cave system. Interaction is accomplished by HBR and a handheld wand with multiple buttons. 

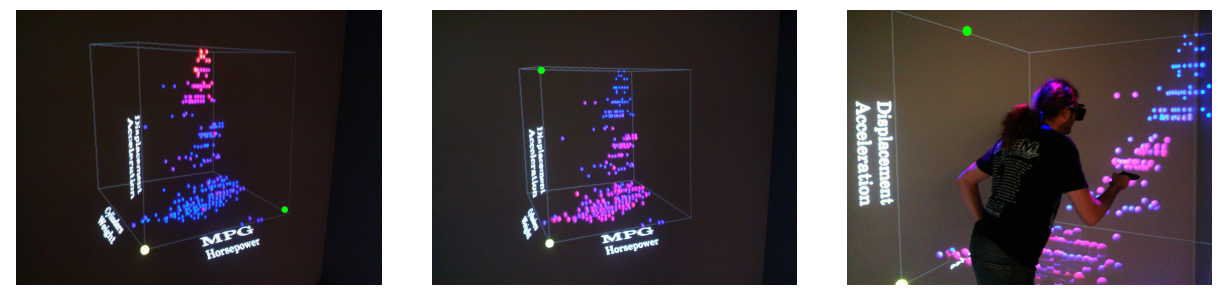

Fig. 3. Size and color of data points within iSP change dependent on user position introducing means to explore a six-dimensional data space (left, center). Unlocking the user position allows the viewer to freely explore interesting set-ups (right, orange point represent last user position).

\subsection{Immersive Scatter Plot (iSP)}

Background and main idea: In common scatter plots dimensions must be neglected when data of higher dimensionality is to be visualized leading to information loss. By taking advantage of immersion and treating the position of the immersed viewer as an individual data point in data space, an immersive Scatter Plot (iSP) is able to represent characteristics of higher-dimensional data. Six-dimensional (6D) data is used in this example.

Representation: An iSP without an immersed viewer is identical to an ordinary 3D scatter plot (see Figure 2, left). In order to represent data of higher dimensionality, we take advantage of the "worlds within worlds" metaphor [17] and overlay the displayed primary coordinate system, the master plot, with a secondary coordinate system, the navigation plot. Both systems share the same origin and alignment of the dimension axes. The master plot represents three dimensions of the data arbitrarily selected by the author via interactive menus. As within the traditional scatter plot, these dimensions determine the respective positions of the data points in the plot. The navigation plot is formed by three other dimensions of the data and serves only for navigation purposes. The corresponding data points are not shown. Both plots are linked by an immersed viewer that is placed inside the visualization. As both plots are superimposed, the position of the viewer determines a point in the $6 \mathrm{D}$ data space. The distance of this point with regard to all other points is then encoded in their size and color (see Figure 3). Distances are calculated in 6D space using the Euclidian distance metric. Data points that are close to the user position are larger and brighter.

Interaction: The viewer mainly interacts with the plot by a constant change of the position and an analysis of the changes that this imposes on the visual encoding of the data points. This makes it possible to evaluate properties, e.g., data clusters, that are observed in the master plot for their behavior in $6 \mathrm{D}$ space. If the cluster points show identical or similar encodings during position changes, the cluster property holds for the $6 \mathrm{D}$ data space (see Figure 3, center). 
Different groups of cluster points identifiable by their respective size and color represent different data clusters in 6D space (see Figure 2, center and right). For a structured exploration of the data space two strategies are proposed: (1) single and (2) multiple axes browsing. In single axis browsing, the user changes position with regard to a single axis giving a feedback about the properties of the data with regard to the associated dimension only (see Figure 3, left). In multiple axes browsing the user position is varied along a certain trajectory in the plot providing feedback to higher-dimensional correlations (see Figure 3, center).

As it is not always desired to track the user position, we implemented the option to disable this option. This keeps the point representation constant allowing for its further exploration at different angles or distances (see Figure 3, right). Although not mandatory for immersion, we also provide an interactive wand making it possible to pan, zoom, or rotate the visualization in presentation space.

Properties: The iSP approach allows to explore a $6 \mathrm{D}$ data set by a common 3D scatter plot. Our tests have shown that it is simple to "mentally" link both plots, although it might take some initial training efforts to understand the principle and to memorize the dimension mapping. As both coordinate systems are superimposed, only a single $3 \mathrm{D}$ position must be determined to extrapolate the associated $6 \mathrm{D}$ point. Position changes allow for a quick and reliable feedback about higher-dimensional data features. The immersion of the viewer and the resulting close proximity to the displayed data points might be a drawback for certain views as it limits the number of points visible within a view. Occlusion of data points can also be an issue. Both problems, however only matter when the mental model of the data is lost. They can be overcome by unlocking the user position from the representation. Further, dimension axes that are overlayed cannot be considered independently. This can be solved by superimposing axes with similar characteristics.
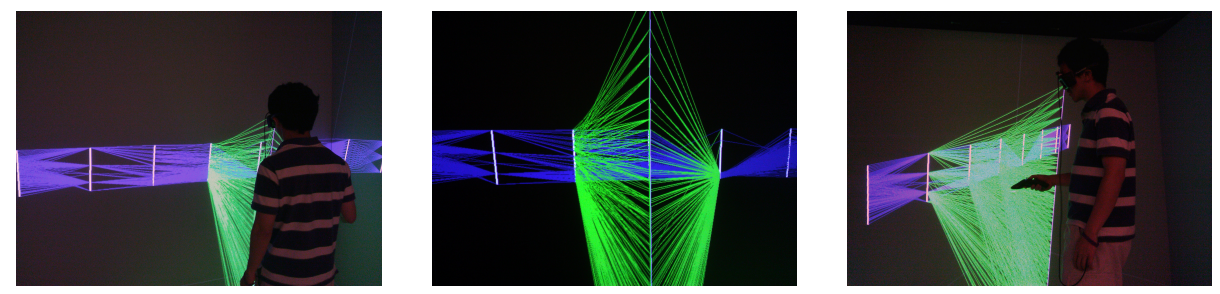

Fig. 4. Immersive Parallel Coordinates consider the user as a central axis that is connected to a specified number of main axes (left, center). In nearest-axes mode, connections change according to user position (right). 


\subsection{Immersive Parallel Coordinates (iPC)}

Background and main idea: There are different $3 \mathrm{D}$ variations of the parallel coordinates plot. Some, such as those described in [18], use the concept of an importance axis that is placed in the center of the representation and connected to all other axes. This can lead to visual clutter for high-dimensional data. User-driven (de)selection of a relevant connections may help to overcome this problem, but interactions are difficult when 2D display technology is used. Immersive Parallel Coordinates (iPC) adopt the approach of a central axis and demonstrate the second concept of iAV - the user serving as part of the layout. $\mathrm{In} \mathrm{iPC}$ the central axis is labeled user axis and represented by the viewer.

Representation: Similar to iSP, the visualization of iPC without an immersed viewer does not significantly differ from its standard counterpart. This especially applies to the main axes that are arranged on a $2 \mathrm{D}$ plane. The user axis is shown as soon as the viewer enters the visualization (Figure 4, left and center) and displayed in front of the viewer stretching from the users top to feet. It is connected to a specified number of main axes.

Interaction: $\mathrm{iPC}$ provide intuitive means for interacting with the representation, again, mostly via position changes. We propose two different interaction modes: (1) nearest-axes and (2) fixed-axes mode. In nearest-axes mode the user axis connects only to a specified number of axes that are closest to the current user position (Figure 4, right). In fixed-axes mode, the connected axes are kept fixed. Changes in user position result in different projections of the data (Figure 5 , left and center). Our tests revealed that during visual analysis the nearestaxes mode is useful to find interesting dimensions in the data. The fixed-axes mode is more suited for a detailed analysis of axes pairs and configurations.

The fact that the user axis is aligned with the user creates a viewing angle to the data that inherently prioritizes lines closer to the eyes of the viewer. To allow for independent views of the data, we provide means similar to that proposed for iSP to disable user tracking (Figure 5, right). We also support wand interaction allowing for different viewing transformations not supported by HBR.
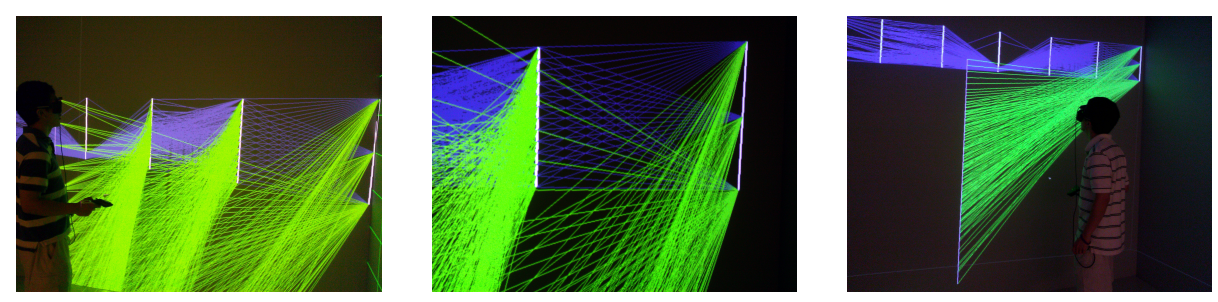

Fig. 5. The fixed-axes mode in iPC allows to reveal patterns in the data by scaling and shearing (left, center). Unlocking the user from the presentation makes it possible to analyze the data from unconstrained viewing angles (right). 
Properties: iPCs allow the viewers to immerse themselves in the layout and to play an active role in data exploration. iPC result in a truly viewer-centered representation. The visualization ensures that the important user axis is always the axis closest to the viewer and thus is perceived in higher detail than any other axis. Changing user position in nearest-axes mode is a natural manner to explore many axes configurations and to browse the visualization dimensionwise. The fixed-axes mode is a means to intuitively distort the representation of the projected data lines by scaling and shearing. In addition to the advanced 3D representation in IEs, this can help to reveal patterns that are not visible in a planar and static representation. As the applied distortion is determined by the respective user position it can be changed effortlessly and is simply understood. We did not observe any disorientation or cybersickness for our implementations of iPC and iSP.

\section{Conclusion and Future Work}

We showed that immersive environments can provide many benefits for the visualization of abstract data. Compared to the common "flat" data display, immersive environments support a highly realistic representation of the three spatial dimensions and natural means to interact in that space. We also introduced a novel concept for the immersive visualization of abstract data involving the user as part of the data or layout. Its implementation has been demonstrated by the adaptation of the scatter and parallel coordinates plot. Both showed the potential of the approach, but represent first attempts in this direction only.

In future research we will evaluate the novel concept by comparisons to established $2 \mathrm{D}$ visualization techniques and comprehensive user tests. We will also be concerned with novel visualization strategies exclusively designed for immersive environments. First developments for cluster visualization based on transient spheres that can be entered in order to provide context for the associated cluster or its sub-clusters are promising. Such visualizations have the potential to lead to a completely novel class of data displays taking full advantage of the benefits provided by immersive environments.

\section{References}

1. Bowman, D., Raja, D., Lucas, J., Datey, A.: Exploring the benefits of immersion for information visualization. In: Proceedings of HCI International. (2005)

2. Lau, H., Chan, L., Wong, R.: A VR-based visualization framework for effective information perception and cognition. In: Proceedings of Human System Interactions. (2008) 203-208

3. Lamm, S.E., Reed, D.A., Scullin, W.H.: Real-Time geographic visualization of world wide web traffic. in proceedings of International World Wide Web conference 28 (1996) 1457-1468

4. Carlo, W.D.: Exploring multi-dimensional remote sensing data with a virtual reality system. Geographical and Environmental Modelling 4 (2000) 7-20 
5. Maletic, J.I., Leigh, J., Marcus, A., Dunlap, G.: Visualizing Object-Oriented software in virtual reality. in Proceedings of the International Workshop on Program Comprehension 26 (2001) 26-35

6. Benford, S., Snowdon, D., Greenhalgh, C., Ingram, R., Knox, I., Brown, C.: VRVIBE: a virtual environment for co-operative information retrieval. Computer Graphics Forum 14 (1995) 349-360

7. Card, S.K., Shneiderman, B.: Readings in Information Visualization - Using Vision to Think. Morgan Kaufman, San Francisco (1999)

8. Fekete, J., Wijk, J.J., Stasko, J.T., North, C.: The value of information visualization. In: Information Visualization: Human-Centered Issues and Perspectives. Springer-Verlag (2008) 1-18

9. Knight, C., Munro, M.: Comprehension with[in] virtual environment visualisations. Proceedings of the IEEE 7th International workshop on program comprehension (1999) 4-11

10. Santos, R.D., Russo, C., Santos, D., Gros, P., Abel, P., Loisel, D., Trichaud, N.: Mapping information onto 3D virtual worlds. in Proceedings of International Conference on Information Visualization (2000) 379-386

11. Kjellin, A., Pettersson, L.W., Seipel, S., Lind, M.: Different levels of 3D: an evaluation of visualized discrete spatiotemporal data in space-time cubes. Information Visualization 9 (2009) 152-164

12. Ware, C., Franck, G.: Evaluating stereo and motion cues for visualizing information nets in three dimensions. ACM Trans. Graph. 15 (1996) 121-140

13. Crossley, M., Davies, N.J., Taylor-Hendry, R.J., McGrath, A.J.: Three-dimensional internet developments. BT Technology Journal 15 (1997)

14. Arns, L., Cruz-Neira, C., Cook, D.: The benefits of statistical visualization in an immersive environment. In: Proceedings of the IEEE Virtual Reality Conference, IEEE Computer Society (1999) 88-95

15. Bowman, D., Lucas, J., North, C., Raja, D.: Exploring the benefits of immersion in abstract information visualization. In: in Proceedings of International Immersive Projection Technology Workshop. (2004)

16. Knight, C., Munro, M.: Mindless visualisations. In: in Proceedings of ERCIM Workshop "User Interfaces for All". (2000)

17. Feiner, S., Beshers, C.: Worlds within worlds : Metaphors for exploring nDimensional virtual worlds. Proceedings of the Symposium on User Interface Software and Technology (1990) 76-83

18. Johansson, J., Forsell, C., Lind, M., Cooper, M.: Perceiving patterns in parallel coordinates: determining thresholds for identification of relationships. Information Visualization 7 (2008) 152-162 